\title{
Group Object Structure and State Estimation with Evolving Networks and Monte Carlo Methods
}

\author{
Amadou Gning ${ }^{1}$, Lyudmila Mihaylova ${ }^{1}$, Simon Maskell $^{2}$, \\ Sze Kim Pang ${ }^{3}$ and Simon Godsill ${ }^{3}$ \\ ${ }^{1}$ Dept of Communication Systems, Lancaster University, UK \\ ${ }^{2}$ QinetiQ, Malvern Technology Centre, Worcestershire, UK \\ ${ }^{3}$ Dept. of Engineering, University of Cambridge, UK \\ \{e.gning,mila.mihaylova\}@lancaster.ac.uk,s.maskell@signal.qinetiq.com, \{skp31,sjg\}@eng.cam.ac.uk
}

\begin{abstract}
This paper proposes a technique for motion estimation of groups of targets based on evolving graph networks. The main novelty over alternative group tracking techniques stems from learning the network structure for the groups. Each node of the graph corresponds to a target within the group. The uncertainty of the group structure is estimated jointly with the group target states. New group structure evolving models are proposed for automatic graph structure initialisation, incorporation of new nodes, unexisting nodes removal and the edge update. Both the state and the graph structure are updated based on range and bearing measurements. This evolving graph model is propagated combined with a sequential Monte Carlo framework able to cope with measurement origin uncertainty. The effectiveness of the proposed approach is illustrated over scenarios for group motion estimation in urban environments. Results with challenging scenarios with merging, splitting and crossing of groups are presented with high estimation accuracy. The performance of the algorithm is also evaluated and shown on real ground moving target indicator (GMTI) radar data and in the presence of data origin uncertainty.
\end{abstract}

Keywords - evolving graphs, random graphs, group target tracking, nonlinear estimation, Monte Carlo methods, Metropolis-Hastings step

\section{INTRODUCTION}

During the last years group object tracking has been investigated in various different applications including road traffic systems, military surveillance and in particular for ground moving target indicator (GMTI) tracking [1] and robotics applications [2]-[5].

Groups of targets can be considered as formations of entities whose number varies over time because targets can enter a scene, or disappear at random times. The groups can split, merge, to be relatively near to each other or move largely independently on each other. However, it is typical for group formations to maintain some patterns of movement [6] and hence the methods for group tracking differ from the methods of

This paper was submitted August 2008, revised October 2009, April 2010 and September 2010. standard multiple-target tracking. Although individual targets in the group can exhibit independent movement at a certain level, overall the group will move as one whole, synchronising the movement of the individual entities and avoiding collisions. In most of the multitarget tracking methods, as opposed to groups tracking methods, tracking of individual objects is the common approach. However, there are strong motivations to model and to study the behaviour of groups. One motivation is the ability to statistically infer which tracks are moving in formation or are having common movement. We may also want to detect events inside groups (splitting) and between groups (merging). This information fits well with a number of modern multitarget tracking applications where one may want to differentiate friendly objects from enemies or to predict the intention, destination and future manoeuvres of targets. Moreover, another strong motivation for group tracking is in the possibility of using common information about the group to improve the estimation of the objects' individual states. For instance, in case of low detection probabilities and/or very noisy environments, by modeling the targets' interactions inside groups, the detection of stealthy targets can be facilitated [7]. A further motivation is that a user may be unable to assimilate information relating to large numbers of individual objects. Group object estimation makes it possible, for such a user, to detect events or focus on particular groups with interesting behaviour. Finally, an additional motivation is to consider common applications where objects have multiple parts, each of which generates detections (e.g. when multiple radar scatters exist in a single extended object). In many cases group object tracking is the only applicable approach, for instance when tracking thousands of targets that may not be possible to be individually tracked [8]. Rescuing people in earthquakes, floods and disaster events also necessitate approaches where the whole group motion is monitored instead of the motion of each individual person. 
In [9] the benefit of group object tracking over individual object tracking is demonstrated over simulated and real data in terms of estimation accuracy. The interactions between group members are modelled by repulsive forces. In these classes of problems, group modeling offers a natural solution.

Different models of groups of objects have been proposed in the literature, such as particle models for flocks of birds [10]-[12], and leader-follower models [6]. However, estimating the dynamic evolution of the group structure has not been widely studied in the literature, although there are similarities with methods used in evolving network models [13], [14].

Methods for group object tracking also vary widely: from Kalman filtering approaches, Joint Probability Data Association (JPDA) [15], [16] to Probability Hypothesis Density (PHD) filtering [17]-[19], and others [20]-[24]. The influence of the 'negative' information on group object tracking is considered in [25] and ground moving target indicator tracking based on particle filtering in [1]. In [19] a coordinated group tracking model is presented, comprising a continuoustime motion of the group and a group structure transition model. A Markov chain Monte Carlo (MCMC) particle filter algorithm is proposed to approximate the posterior probability density function (PDF) of the high dimensional state.

Mahler [6] outlines that careful group target motion models should be able to describe target appearance and disappearance, not just for the motion of individual targets and the degree to which targets jointly move in a coordinated manner.

Inspired by some ideas from [13], we consider the groups of objects as evolving undirected random graphs. The novelty of this paper is in the proposed approach for estimating the group structure jointly with the group target states using a graphical representation. With this graphical representation, objects are not assigned to groups but are connected to one another. This enables the cohesion of a group to be precisely modeled. The main contributions for this work consist in: i) the developed graphical representation of the group structure, ii) a second graphical model is developed for the groups which gives information about mutually interacting groups and that is also used in the data association algorithm, iii) finally, target state estimates (from the designed Monte Carlo methods) within the same group or within interacting groups are compared in order to update the graph.

The remaining parts of this paper are organised as follows. Section II presents the evolving network models. Section IV formulates the group object tracking problem jointly with the proposed evolving network model for the groups. Section VI presents results with simulated data and from real GMTI radar measurements, with measurement origin uncertainty. Finally, conclusions are given in Section VII.

\section{Evolving Network Models}

The evolution of complex network structures has been studied in the light of different problems, such as complex networks in communications, biology, social sciences, economics and Internet (see, e.g., the surveys [13], [14]). Graph theory represents natural ways of modeling these network structures. Within this graphical family, random graphs introduced in the early sixty by Erdos and Renyi [26] are the first approach attempting to model these complex evolving networks. A random graph of size $n$ is simply obtained by starting with a set of $n$ vertices and by adding randomly edges between them. In the first model proposed in [26], every possible edge in the graph occurs with a chosen common probability. After several studies and generalisation on random graph network theory, recent research in networks has been focussed on more sophisticated evolving dynamic systems. The main difference stems from the necessity to continuously change the size of the graph (e.g., due to addition of new nodes or removal of nodes). Another major difference is in the probabilities associated to the creation of new edges. For instance, when adding a new node, instead of using a random process with an equal probability for the generation of new edges, a preferential creation of edges can be computed. The preferential strategy of adding edges is based on the assumption that a node with a higher impact in the graph network has a higher probability to be connected to new nodes than a second node with less impact. For instance, for a research community network, an article with many citations has more chances to be cited than a paper with few citations.

The flexible approach of evolving graphs fits well to the problem of group object tracking. The closest application to the group modelling task is the WorldWide Web (WWW) network representing a large dynamic network where nodes and links are continuously created and removed [13]. However, the network characterising the group object evolution is obviously more dynamic than the WWW network where the effects of removed links between nodes are often negligible. A significant novelty in the evolving group object network that we develop is that the targets have dynamical spatial constraints. The preferential approach is consequently irrelevant for the group tracking problem and more appropriate evolving models need to be introduced.

In this paper we extend concepts of evolving network models to group object network in Section III. A graphical representation models the connections between targets. At each time step new nodes are added, existing nodes are removed and the set of edges is updated.

\section{An Evolving Network MOdel fOR Group MOTION ESTIMATION}

One of the challenges in group object tracking is in the necessity of updating the group structure and mod- 
eling the interactions between separate components. For this purpose adding components to the groups, removing others, splitting and merging groups are of primary importance.

In our paper, $\boldsymbol{G}_{t}$ is chosen to be an evolving undirected random graph representing both the targets within the groups (nodes in the graph) and some relations between the group members, which is reflected by the edges between the related graph nodes. Symmetric criteria as distance and velocity are used to create an edge. However, other criteria can be applied.

\section{A. Graphical Representation for the Group Object Structure}

Consider $N$ targets constituting the set of vertices $\left\{\boldsymbol{v}_{1}, \ldots, \boldsymbol{v}_{N}\right\}$. Each vertex $\boldsymbol{v}_{i}$ is associated with the target state and with the target state's corresponding variance. The set of edges linking the set of vertices is denoted by $E$. The graph structure can then be denoted by $\boldsymbol{G}=\left(\left\{\boldsymbol{v}_{1}, \ldots, \boldsymbol{v}_{N}\right\}, E\right)$. One edge, in $E$, between two nodes $\boldsymbol{v}_{i}$ and $\boldsymbol{v}_{j}$ is denoted by $\left(\boldsymbol{v}_{i}, \boldsymbol{v}_{j}\right)$. In order to characterise the presence or absence of a link (edge) between two nodes, the distance between these two considered nodes is calculated, e.g., by the Mahalanobis distance criterion. The Mahalanobis distance is computed from the estimated positions and from the velocities of the separate objects. This estimated distance is thresholded and a decision is made about the connections. In this representation a group corresponds to a connected component of the graph structure. Note that, two nodes are in the same connected component if and only if a path between them exists. In the following sections, the groups in $\boldsymbol{G}_{t}$ are denoted $\left\{\boldsymbol{g}_{1}, \ldots, \boldsymbol{g}_{n_{G}}\right\}$, where the groups $\boldsymbol{g}_{i}$ are the connected components of $\boldsymbol{G}$ and $n_{G}$ is the number of groups in $\boldsymbol{G}$.

In [19], $\boldsymbol{G}_{t}$ represents a set of group's labels for each target. For example, with five targets, $\boldsymbol{G}_{t}=\left[\begin{array}{llll}1 & 1 & 2 & 2\end{array}\right]$ means that targets 1 and 2 are in group 1 and targets 3,4 and 5 are in group 2. With the graphical representation, one similar group structure is: $\boldsymbol{G}_{t}=$ $\left(\left\{\boldsymbol{v}_{1}, \boldsymbol{v}_{2}, \boldsymbol{v}_{3}, \boldsymbol{v}_{4}, \boldsymbol{v}_{5}\right\}, \quad\left\{\left(\boldsymbol{v}_{1}, \boldsymbol{v}_{2}\right),\left(\boldsymbol{v}_{3}, \boldsymbol{v}_{4}\right),\left(\boldsymbol{v}_{3}, \boldsymbol{v}_{5}\right)\right\}\right)$ and the groups correspond to the connected components of the graph $G_{t}$.

\section{B. Motivations for the Group Object Structure Graph- ical Representation}

The approach proposed in this paper builds up a dynamical evolution model instead of using transition probabilities in the space of possible group structures (e.g., see Figure 1).

Algorithms of adding components to the groups, removing others, splitting and merging groups by taking into account geometric distances and velocity distances between the groups and between the targets are proposed.

In [19] the approach with transition probabilities is followed. In contrast with [19], an evolution model

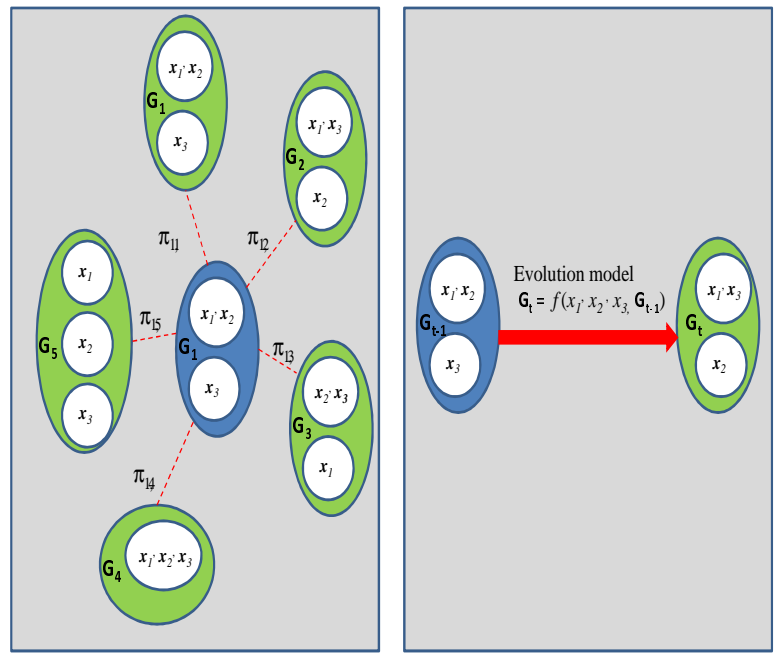

Fig. 1. Two approaches for modeling dynamical changes on the graph structure. At left: transition probabilities $\pi_{1, j}, j=1, \ldots, 5$ in the space of possible group structures (built, for this example, from 3 existing targets with respective states: $\boldsymbol{x}_{1}, \boldsymbol{x}_{2}$ and $\left.\boldsymbol{x}_{3}\right)$. At right: an evolution model for $\boldsymbol{G}_{t}$ according to the previous graph structure $\boldsymbol{G}_{t-1}$ and according to the current states $\boldsymbol{x}_{1}, \boldsymbol{x}_{2}$ and $\boldsymbol{x}_{3}$ of all targets. Bold (blue) ellipses denote the current group structure, the others ellipses (light-green) denote new group structures that may be reached in one time step.

is designed for the group structure by incorporating the information about closeness between the groups and about closeness between targets within a group, in a graphical way. At each time instant, based on the decision made about birth and death targets, nodes are created or removed inside a group. For each removed node, all its links to other nodes are deleted, and for each new node, respective links to neighbour nodes are added. Similarly, when an object passes from one group to another, the respective links (edges) in the considered graph disappear, and one or more links will appear in the graph of the other group which the object joins.

A strong motivation for such graphical representation is illustrated in Figure 2. The graphical representation allows an easy switch in the group structure space: removing or adding only one edge can change the group structure.

A further motivation is illustrated in Figure 3 which shows two groups $\boldsymbol{g}_{1}$ and $\boldsymbol{g}_{2}$ with the same nodes $\left\{\boldsymbol{v}_{1}, \ldots, \boldsymbol{v}_{4}\right\}$. These two groups are identical if considered as a set of indexes. When propagating these two groups, using the graph representation, $\boldsymbol{g}_{2}$ is more likely to split than $\boldsymbol{g}_{1}$. The graph representation, allows, thus, to propagate more information than a vector of group indexes for each target.

\section{Evolving Graph Models}

The aim is to determine an evolution model $\boldsymbol{G}_{t}=$ $f\left(\boldsymbol{G}_{t-1}, \boldsymbol{X}_{t}\right)$ for the group structure, for time $t>0$ and an initialisation process $\boldsymbol{G}_{0}=f\left(\boldsymbol{X}_{0}\right)$ for $t=0$. 
The vector $\boldsymbol{X}_{t}=\left(\boldsymbol{x}_{t, 1}, \ldots, \boldsymbol{x}_{t, n}\right)$ comprises the state vectors of all the targets and $f$ denotes the desired evolution model.

The system

$$
\left\{\begin{aligned}
t=0, & \boldsymbol{G}_{0}=f_{I}\left(\boldsymbol{X}_{0}\right), \\
t>0, \boldsymbol{G}_{t} & =f_{N S} \circ f_{N I} \circ f_{E U}\left(\boldsymbol{G}_{t-1}, \boldsymbol{X}_{t}\right),
\end{aligned}\right.
$$

shows the decomposition of the evolution model $f$ according to the time $t$ and according to three distinctive steps: edge update, node incorporation and node removal where $\circ$ denotes the composition operation; $f_{I}$ is an Initialisation model that will be defined in Section III-D; $f_{E U}$ is the graph Edge Updating model that will be defined in Section III-E; $f_{N I}$ is the graph Nodes

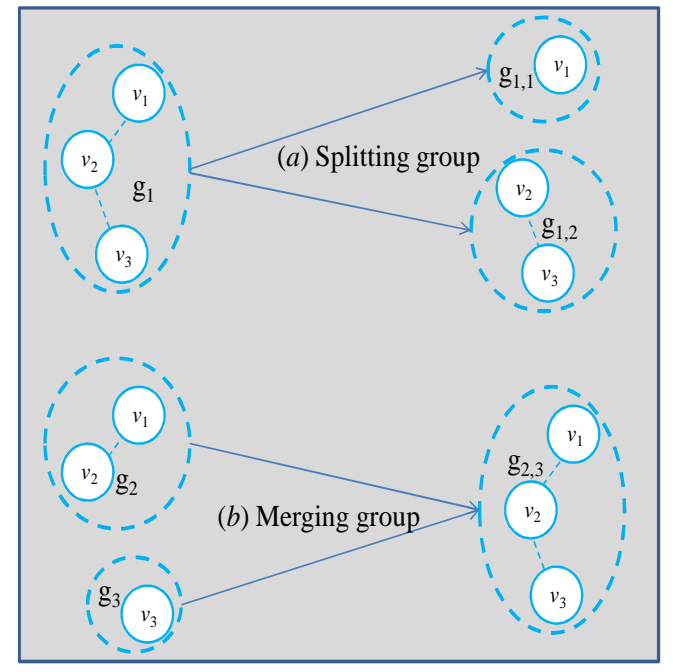

Fig. 2. One strong motivation of using a graphical representation. In this example, with 3 nodes, in $(a)$ a simple removal of one edge can model a splitting group $\boldsymbol{g}_{1}$ into 2 groups $\boldsymbol{g}_{1,1}$ and $\boldsymbol{g}_{1,2}$. In $(b)$, in contrast with $(a)$, one new edge can model merging of two groups $\boldsymbol{g}_{2}$ and $\boldsymbol{g}_{3}$ in one new group $\boldsymbol{g}_{2,3}$

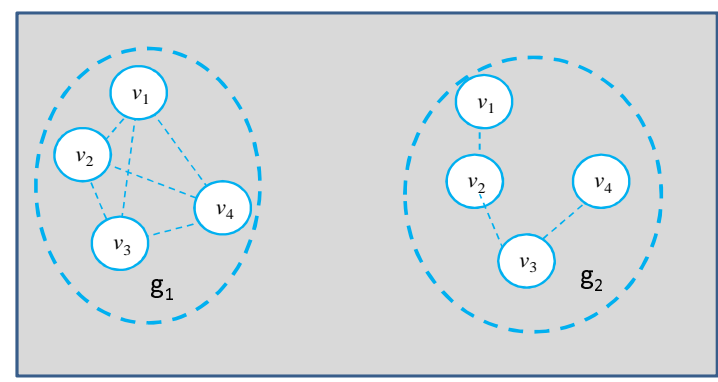

Fig. 3. Motivation of using a graphical representation. In this example, with 4 nodes, 2 graphs represent 2 groups. These two groups are identical if considered as a set of indexes. At left, the graph representing $\boldsymbol{g}_{1}$ contains more edges than the one, at right, representing $\boldsymbol{g}_{2}: \boldsymbol{g}_{2}$ is more likely to split than $\boldsymbol{g}_{1}$.
Incorporation model that will be defined in Section III-F; $f_{N S}$ is the graph Nodes Suppression model that will be defined in Section III-G.

\section{Graph Initialisation- Model $f_{I}$}

In this Section, we assume that, at time $t=0$, the number of targets and their respective states are known, given by one of the detection techniques from [16]. Let us consider $N$ targets constituting the set of vertices $\left\{\boldsymbol{v}_{1}, \ldots, \boldsymbol{v}_{N}\right\}$. Each vertex $\boldsymbol{v}_{i}$ is associated with the target state $\boldsymbol{x}_{0, i}$ at time $t=0$, as well as the target state's corresponding variance matrix $\boldsymbol{P}_{0, i}$. Model 1, given below describes the proposed edge initialisation method where $E_{0}$ is the set of edges linking the set of vertices $\left\{\boldsymbol{v}_{1}, \ldots, \boldsymbol{v}_{N}\right\}$. Initially $E_{0}$ is the empty set $\{\varnothing\}$. The Mahalanobis distance $d_{i, k}$ between vertices $\boldsymbol{v}_{i}$ and $\boldsymbol{v}_{k}$ is calculated and we evaluate whether it exceeds a chosen decision threshold $\varepsilon$. The edge between nodes $\boldsymbol{v}_{i}$ and $\boldsymbol{v}_{k}$ is denoted by $(i, k)$. Using Model 1,

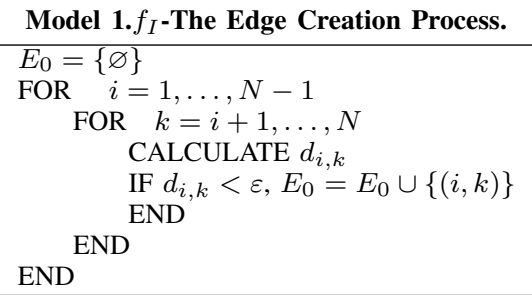

the initial graph structure $\boldsymbol{G}_{0}=\left(\left\{\boldsymbol{v}_{1}, \ldots, \boldsymbol{v}_{N}\right\}, E_{0}\right)$ is then obtained.

\section{E. Edge Updating-Model $f_{E U}$}

The evolving graph of group of targets is more dynamic than those studied in the literature [13]. Existing edges should be updated at each time instant since the graph structure is related with the dynamic spatial configuration. In a straightforward way, Model 1 can recalculate the distance between any pair of nodes. However, the computational complexity can be reduced when some information about group centres (means, covariances and the distances between them) is used. For each group $\boldsymbol{g}$ we define its centre $\boldsymbol{O}^{\boldsymbol{g}}=\frac{1}{n_{g}} \sum_{\boldsymbol{v}_{k} \in \boldsymbol{g}} \boldsymbol{x}_{k}^{\boldsymbol{g}}$ and its corresponding average covariance matrix $\boldsymbol{P}^{g}=$ $\frac{1}{n_{g}} \sum_{v_{k} \in \boldsymbol{g}} \boldsymbol{P}_{k}^{g}$ where $n_{g}$ is defined as the number of targets in $\boldsymbol{g}$. The centre and covariance matrix of each group can be characterised differently, e.g., based on a mixture of Gaussian components.

Using the Mahalanobis distance criterion, an appropriate threshold $\varepsilon^{\prime}>\varepsilon$, and based on Model 1 , a second graph $\boldsymbol{G}^{\prime}=\left(\left\{\boldsymbol{v}_{1}^{\prime}, \ldots, \boldsymbol{v}_{n_{G}}^{\prime}\right\}, E^{\prime}\right)$ can be introduced with nodes $\boldsymbol{v}_{i}^{\prime}$ being characterised by their position $O^{g_{i}}$. A couple of connected nodes in the set $E^{\prime}$ can be interpreted as two groups that can possibly have interactions (exchange of targets). Model 2 summarises the edge updating process between neighbouring groups. The graph $G^{\prime}$ will also be used in the node incorporation process. 
Model 2. $f_{E U}$-Edges Updating Process.

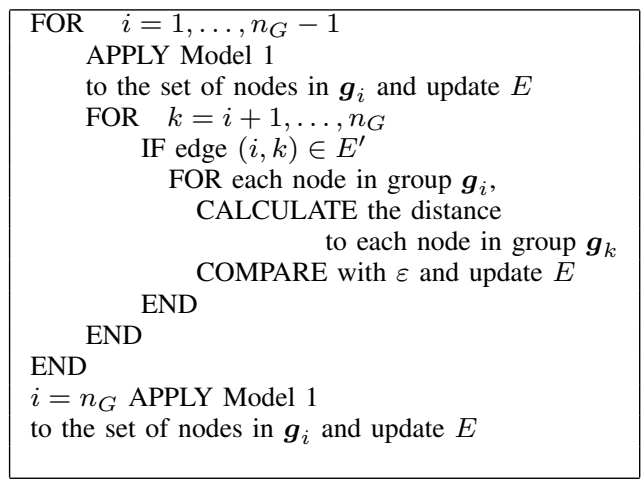

Model 2 can be illustrated using the example from Figure 4. The considered graph contains 3 groups of 12 nodes. In Figure $4(a)$, by introducing the centre of each group, the graph $G^{\prime}$ is represented: it contains 3 nodes, corresponding to the centre of each group, and one edge between $\boldsymbol{g}_{1}$ and $\boldsymbol{g}_{2}$. Figure $4(b)$ and $(c)$ illustrates the update of Model 2.

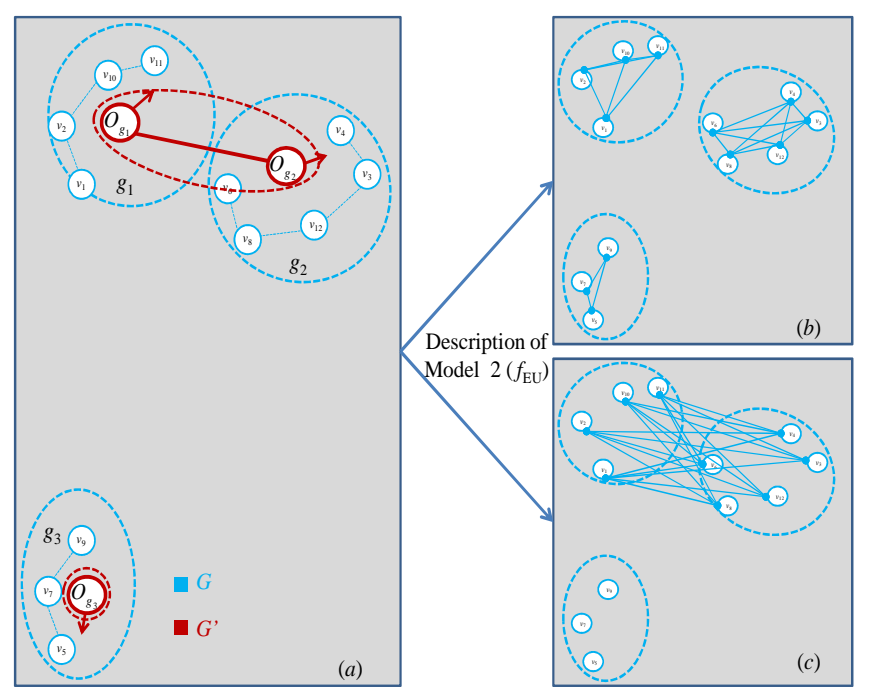

Fig. 4. Model 2: (a) use a second graph structure $\boldsymbol{G}^{\prime}$ and, for the edge updating process, $(b)$ calculate distances between nodes in the same group and (c) calculate distances between nodes in groups that are connected through $G^{\prime}$.

In each group, distances between any couple of nodes are calculated as shown in Figure $4(b)$. Furthermore, in Figure $4(c)$, for any couple of groups $\left(\boldsymbol{g}_{i}, \boldsymbol{g}_{j}\right)$ connected in graph $\boldsymbol{G}^{\prime}$ (in this example, only $\boldsymbol{g}_{1}$ and $\boldsymbol{g}_{2}$ are connected). The distances between any couple of nodes $\left(\boldsymbol{v}_{i}, \boldsymbol{v}_{j}\right)$, chosen respectively in groups $\boldsymbol{g}_{i}$ and $\boldsymbol{g}_{j}$, are calculated. The use of Model 2, in this example, avoids calculations of distances between nodes in $\boldsymbol{g}_{3}$ and nodes in $\boldsymbol{g}_{1}$ and $\boldsymbol{g}_{2}$, respectively.

\section{F. New Node Incorporation-Model $f_{N I}$}

Classical approaches rely on either random or preferential approaches (the mixture of the two also exists) in order to assign edges to the new nodes. Additionally, in classical graph techniques, the number of new edges assigned to each new node is fixed. The approach proposed in this paper differs from the above mentioned techniques. For the purposes of group tracking, the

Model 3. $f_{N I}$ - Incorporation of new nodes.

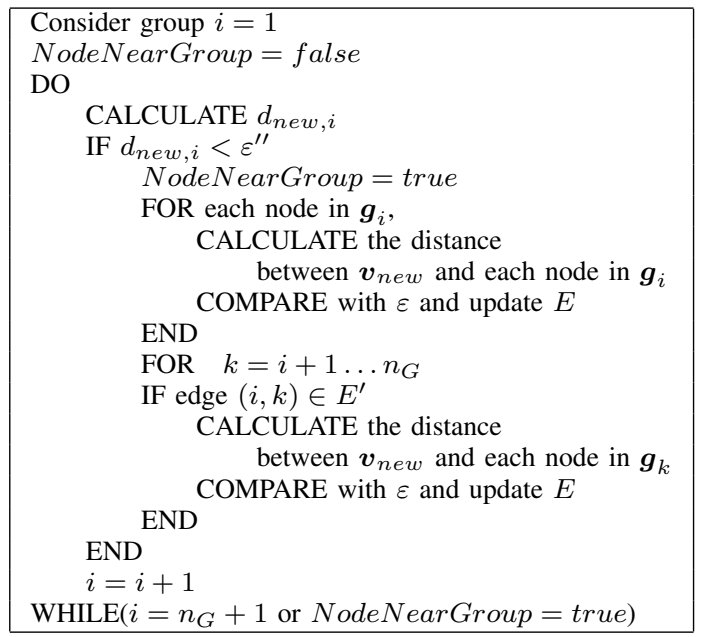

distance calculated based on the interaction criterion should be used to create edges with the existing nodes and the number of edges is then determined by the nodes' spatial configuration. Consider a new node (vertex) denoted as $\boldsymbol{v}_{\text {new }}$ and its state $\boldsymbol{x}_{n e w}$. Depending on the state $\boldsymbol{x}_{n e w}$ and in comparison with the existing $n_{G}$ nodes, new edges have to be created. A simple way is to evaluate the criterion for the interaction between every pair $\left(\boldsymbol{v}_{\text {new }}, \boldsymbol{v}_{i}\right)$. In order to optimise the computational time, the graph $G^{\prime}$ defined in Section III-E can be used.

Model 3 shows the edge updating process when incorporating a new node, where $d_{\text {new }, i}$ is the Mahalanobis distance between $\boldsymbol{v}_{\text {new }}$ and $\boldsymbol{O}_{g_{i}}\left(d_{\text {new }, i}=\right.$ Mahalanobis-distance $\left(\left(\boldsymbol{x}_{n e w}, \boldsymbol{P}_{n e w}\right),\left(\frac{1}{n_{g_{i}}} \sum_{\boldsymbol{v}_{k} \in \boldsymbol{g}_{\boldsymbol{i}}} \boldsymbol{x}_{k}^{\boldsymbol{g}_{\boldsymbol{i}}}\right.\right.$ $\left.\frac{1}{n_{g_{i}}} \sum_{\boldsymbol{v}_{k} \in \boldsymbol{g}_{\boldsymbol{i}}} \boldsymbol{P}_{k}^{\boldsymbol{g}_{\boldsymbol{i}}}\right)$ ); the fixed threshold $\varepsilon^{\prime \prime}>\varepsilon$ introduced in order to see whether the new node $\boldsymbol{v}_{n e w}$ is interacting with a node in a group $\boldsymbol{g}$.

Let us illustrate Model 3 using the example from Figure 5. The considered graph contains 4 groups of 14 nodes. In Figure $5(a)$, by introducing the centre of each group, the graph $G^{\prime}$ is represented: it contains 4 nodes, corresponding to the centre of each group, and two edges between, respectively, $\boldsymbol{g}_{1}$ and $\boldsymbol{g}_{2}$ and $\boldsymbol{g}_{3}$ and $\boldsymbol{g}_{4}$. Distances $d_{n e w, i}$ between the new node $\boldsymbol{v}_{\text {new }}$ and centres of groups $\boldsymbol{O}_{i}$ are computed. The principle of Model 3 is to calculate distances $d_{n e w, i}$ until finding one neighbour group of node $\boldsymbol{v}_{\text {new }}$ according to a threshold $\varepsilon^{\prime \prime}$ or until reaching the last index $i\left(i=n_{G}\right)$. Note that $\varepsilon^{\prime \prime}$ is chosen such that $\varepsilon^{\prime \prime}<<\varepsilon^{\prime}$ so that a new node close to one group $\boldsymbol{g}$ according the threshold $\varepsilon^{\prime \prime}$ is far from any group that is not connected with $g$ according to the threshold $\varepsilon^{\prime}$.

For the example presented on Figure 5, $\boldsymbol{g}_{1}$ and $\boldsymbol{g}_{2}$ are not neighbours of $\boldsymbol{v}_{\text {new }}$ according to the distance criterion. In contrast, $\boldsymbol{g}_{3}$ satisfies the distance criterion. Then, the calculated distances of Model 3, used to update graph $\boldsymbol{G}$, are illustrated. Distances between 


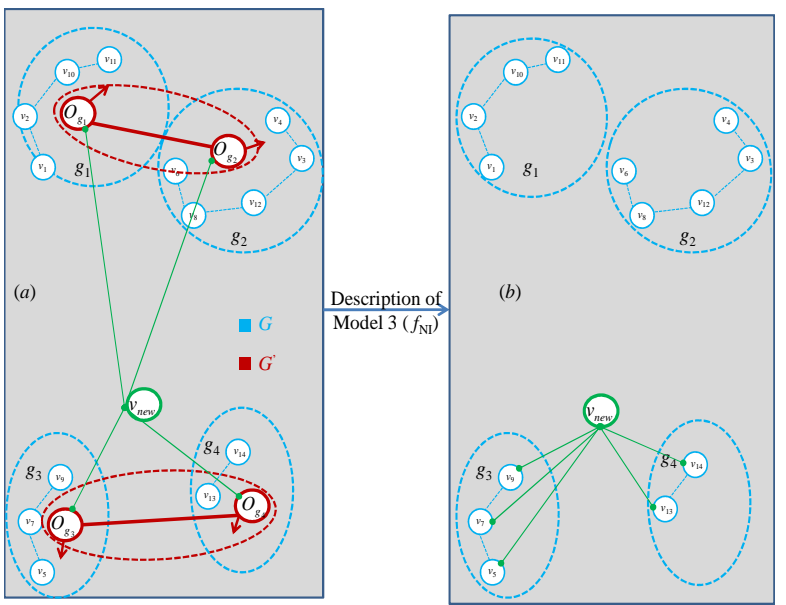

Fig. 5. Model 3: (a) use graph structure $G^{\prime}$ by calculating the distance from the new node $\boldsymbol{v}_{\text {new }}$ to the centres of all groups. Once one group $\boldsymbol{g}_{\boldsymbol{i}}$ satisfies the distance threshold, (b) calculate the distances between $\boldsymbol{v}_{n e w}$ and any node in $\boldsymbol{g}_{\boldsymbol{i}}$ in addition to the distance between $\boldsymbol{v}_{\text {new }}$ and any node in a group connected to $\boldsymbol{g}_{\boldsymbol{i}}$ through $G^{\prime}$.

$\boldsymbol{v}_{n e w}$ and any node in $\boldsymbol{g}_{3}$ are calculated as shown in Figure $5(b)$. Furthermore, since $\boldsymbol{g}_{4}$ is connected to group $\boldsymbol{g}_{3}$, in graph $\boldsymbol{G}^{\prime}$, distances between $\boldsymbol{v}_{\text {new }}$ and any node in $\boldsymbol{g}_{4}$ are also calculated.

The use of Model 3, in this example, avoids the calculation of distances between $\boldsymbol{v}_{\text {new }}$ and nodes in $\boldsymbol{g}_{1}$ and $\boldsymbol{g}_{2}$, respectively.

\section{G. Old Node Suppression-Model $f_{N S}$}

This is the simplest graphical evolution modeling part and consists of removing death targets by removing corresponding nodes and their related edges. A target in the graph will be removed if the measurements do not contain any information about it after a certain period of time.

\section{PRoblem Formulation}

Consider the problem of tracking the motion of groups of targets. Each target $i$ is characterised by its state vector $\boldsymbol{x}_{t, i}=\left(x_{t, i}, \dot{x}_{t, i}, y_{t, i}, \dot{y}_{t, i}\right)^{\prime}$ (comprising the positions $x_{t, i}, y_{t, i}$ and velocities $\dot{x}_{t, i}, \dot{y}_{t, i}$ in $x$ and $y$ directions respectively); ' denotes the transpose operation. Targets which are close to one another tend to form a group. The Mahalanobis distance $d_{i, k}$ is chosen as a criterion of closeness between the targets within a group. At each time instant $t$, the set of objects tracked in a group $\boldsymbol{g}$ can be modeled by a Random Finite Set (RFS, see [6]) that incorporates the state vectors of the group members, $\boldsymbol{X}_{t}^{\boldsymbol{g}}=\left\{\boldsymbol{x}^{\boldsymbol{g}}{ }_{t, 1}, \boldsymbol{x}_{t, 2}^{\boldsymbol{g}}, \ldots, \boldsymbol{x}_{t, n_{g}}\right\}\left(n_{g}\right.$ is the random size of group $\boldsymbol{g}$ ). Knowing the group structure $\boldsymbol{G}_{t}=\left\{\boldsymbol{g}_{1}, \ldots, \boldsymbol{g}_{n_{G}}\right\}$ ( $n_{G}$ is the number of groups), the joint state for the all the targets in the $n_{G}$ groups has the expression $\boldsymbol{X}_{t}=\left\{\boldsymbol{X}_{t}^{\boldsymbol{g}_{1}}, \ldots, \boldsymbol{X}_{t}^{\boldsymbol{g}_{n_{G}}}\right\}$.

At time $t$ a measurement vector $\boldsymbol{z}_{t}$ is received which can be described as a function of the state $\boldsymbol{X}_{t}=$ $\left\{\boldsymbol{X}_{t}^{\boldsymbol{g}_{1}}, \ldots, \boldsymbol{X}_{t}^{\boldsymbol{g}_{n_{G}}}\right\}$. Assuming that the measurement likelihood function $p\left(\boldsymbol{z}_{t} \mid \boldsymbol{X}_{t}\right)$ can be calculated, the purpose is to compute sequentially the state PDF for each group of objects. The changes of the groups such as merging and splitting are taken into account during the graph update process. Additionally, the groups' movements are assumed independent.

Under the Markovian assumption for the state transition, the Bayesian prediction and filtering steps can be written as follows:

$$
\begin{gathered}
p\left(\boldsymbol{X}_{t}, \boldsymbol{G}_{t} \mid \boldsymbol{Z}_{1: t-1}\right)=p\left(\boldsymbol{G}_{t} \mid \boldsymbol{X}_{t}, \boldsymbol{Z}_{1: t-1}\right) \times p\left(\boldsymbol{X}_{t} \mid \boldsymbol{Z}_{1: t-1}\right) \\
=\int p\left(\boldsymbol{G}_{t} \mid \boldsymbol{X}_{t}, \boldsymbol{G}_{t-1}\right) \times \\
p\left(\boldsymbol{X}_{t} \mid \boldsymbol{X}_{t-1}, \boldsymbol{G}_{t-1}\right) p\left(\boldsymbol{X}_{t-1}, \boldsymbol{G}_{t-1} \mid \boldsymbol{Z}_{1: t-1}\right) d \boldsymbol{X}_{t-1} d \boldsymbol{G}_{t-1}, \\
p\left(\boldsymbol{X}_{t}, \boldsymbol{G}_{t} \mid \boldsymbol{Z}_{1: t}\right)=\frac{p\left(\boldsymbol{z}_{t} \mid \boldsymbol{X}_{t}, \boldsymbol{G}_{t}\right) \times p\left(\boldsymbol{X}_{t}, \boldsymbol{G}_{t} \mid \boldsymbol{Z}_{1: t-1}\right)}{p\left(\boldsymbol{z}_{t} \mid \boldsymbol{Z}_{1: t-1}\right)},
\end{gathered}
$$

where $Z_{1: t}$ is the set of measurements up to time $t$ and $z_{t}$ is the current vector of measurements.

The transition PDF $p\left(\boldsymbol{G}_{t} \mid \boldsymbol{X}_{t}, \boldsymbol{G}_{t-1}\right)$ of the group structure can be calculated knowing the prediction of the target state and group structure in the previous time instant, and using the graph evolution model introduced in Section III-C. The transition PDF $p\left(\boldsymbol{X}_{t} \mid \boldsymbol{X}_{t-1}, \boldsymbol{G}_{t-1}\right)$ of the state of all targets is calculated knowing the previous time target states and group structure PDF $p\left(\boldsymbol{X}_{t-1}, \boldsymbol{G}_{t-1} \mid \boldsymbol{Z}_{1: t-1}\right)$. With the assumption of independence between group motions, the PDF $p\left(\boldsymbol{X}_{t} \mid \boldsymbol{X}_{t-1}, \boldsymbol{G}_{t-1}\right)$ can be decomposed in the following supplementary equation

$$
p\left(\boldsymbol{X}_{t} \mid \boldsymbol{X}_{t-1}, \boldsymbol{G}_{t-1}\right)=\prod_{\boldsymbol{g}_{i} \in \boldsymbol{G}_{t-1}} p\left(\boldsymbol{X}_{t}^{\boldsymbol{g}_{i}} \mid \boldsymbol{X}_{t-1}^{\boldsymbol{g}_{i}}\right),
$$

where $p\left(\boldsymbol{X}_{t}^{\boldsymbol{g}_{i}} \mid \boldsymbol{X}_{t-1}^{\boldsymbol{g}_{i}}\right)$ is the transition density of the set of targets from the group $\boldsymbol{g}_{i}$.

In order to perform the correction step, the likelihood function $p\left(\boldsymbol{z}_{t} \mid \boldsymbol{X}_{t}, \boldsymbol{G}_{t}\right)$ of the whole state has to be evaluated by means of a data association approach. In this paper, the JPDA algorithm [16] is used to resolve the measurement origin uncertainty.

In addition, in the gating process in the JPDA algorithm is enhanced by using other information about the graph structure, such as the distance between groups. Figure 5 shows an example where groups $\boldsymbol{g}_{3}$ and $\boldsymbol{g}_{4}$ can be considered separately from groups $\boldsymbol{g}_{1}$ and $\boldsymbol{g}_{2}$. Note that the graph $\boldsymbol{G}_{t}^{\prime}$ estimated at each time instant is applied in the edge updating process and in the nodes incorporation steps which leads to reduction of the data association computations. At each time step, the graph $G_{t}^{\prime}$ can also be used in the gating process. Indeed groups of the same graph $G_{t}^{\prime}$ 's connected component can be gathered in separate data association process: the graph $G_{t}^{\prime}$ offers a straightforward method of clustering the targets for the data association process.

Denote by $\left\{\boldsymbol{g}_{1}^{\prime}, \ldots, \boldsymbol{g}_{n_{G^{\prime}}}^{\prime}\right\}$, the set of $n_{G^{\prime}}$ connected components in graph $G^{\prime}$. Any connected component $\boldsymbol{g}_{i}^{\prime}$ can model a set of groups that are close enough 
to be treated in the same data association algorithm. Under the independence assumption between the $\boldsymbol{g}_{i}^{\prime}$, the following equation (5) can be written

$$
\begin{gathered}
p\left(\boldsymbol{z}_{t} \mid \boldsymbol{X}_{t}, \boldsymbol{G}_{t}\right)=p\left(\boldsymbol{z}_{t} \mid \boldsymbol{X}_{t}, \boldsymbol{G}_{t}, \boldsymbol{G}_{t}^{\prime}\right) \\
=\prod_{i=1, \ldots, n_{G^{\prime}}} p\left(\boldsymbol{z}_{t}^{\boldsymbol{g}^{\prime}} \mid \boldsymbol{X}_{t-1}^{\boldsymbol{g}_{i}^{\prime}}\right),
\end{gathered}
$$

where $\boldsymbol{X}_{t-1}^{\boldsymbol{g}_{\boldsymbol{i}}^{\prime}}$ is the set of targets' states belonging to the groups in $\boldsymbol{g}_{i}^{\prime}$. The vector $\boldsymbol{z}_{t}^{\boldsymbol{g}_{i}}$ comprises the subset of measurements related with the group in $\boldsymbol{g}_{i}^{\prime}$. For example, $\boldsymbol{z}_{t}^{\boldsymbol{g}_{i}}$ can be chosen by gating measurements using the set of targets state $\boldsymbol{X}_{t-1}^{\boldsymbol{g}_{i}^{\prime}}$.

\section{A. Model of Individual Targets}

The nearly constant velocity model [27], [28] is used for the update of each node of the graph, i.e., for modelling the motion of each target within a group. In two dimensions, the state of the $i$ th target is given by:

$$
\boldsymbol{x}_{t, i}=\boldsymbol{A} \boldsymbol{x}_{t-1, i}+\boldsymbol{\Gamma} \boldsymbol{\eta}_{t-1}
$$

where $\boldsymbol{A}=\operatorname{diag}\left(\boldsymbol{A}_{1}, \boldsymbol{A}_{1}\right), \boldsymbol{A}_{1}=\left(\begin{array}{cc}1 & T \\ 0 & 1\end{array}\right), \boldsymbol{\Gamma}=$ $\left(\begin{array}{cccc}T / 2 & 1 & 0 & 0 \\ 0 & 0 & T / 2 & 1\end{array}\right)^{\prime}, T$ is the sampling interval and $\boldsymbol{\eta}_{t-1}$ is the system dynamics noise. In order to cover a wide range of motions, the velocity should be approximately constant over straight line trajectories and the velocity change should be abrupt at each turn (especially for the direction of the velocity). Then, the system dynamics noise $\boldsymbol{\eta}_{t-1}$ is represented as a sum of two Gaussian components

$$
p\left(\boldsymbol{\eta}_{t-1}\right)=\alpha \mathcal{N}\left(0, \boldsymbol{Q}_{1}\right)+(1-\alpha) \mathcal{N}\left(0, \boldsymbol{Q}_{2}\right),
$$

$\boldsymbol{Q}_{1}=\operatorname{diag}\left(\sigma^{2}, \sigma_{1}^{2}\right), \boldsymbol{Q}_{2}=\operatorname{diag}\left(\sigma^{2}, \sigma_{2}^{2}\right) ; \sigma$ is a standard deviation assumed common and constant for $x$ and $y$; $\sigma_{1} \ll \sigma_{2}$ are standard deviations allowing to model respectively smooth and abrupt changes in the velocity. The fixed coefficient $\alpha$ has values in the interval $[0,1]$.

In addition, to model the interaction between objects in each group, the average velocity of group objects is used in (6) instead of the velocity of each group component. For each group $\boldsymbol{g}$, in the group structure $\boldsymbol{G}$

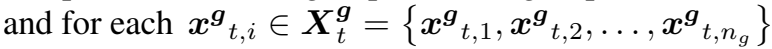
we have the following equation

$$
\boldsymbol{x}_{t, i}^{\boldsymbol{g}}=\boldsymbol{x}_{t-1, i}^{\boldsymbol{g}}+\sum_{j=1}^{n_{g}}\left(\boldsymbol{B} \boldsymbol{x}_{t-1, j}^{\boldsymbol{g}}\right) \quad+\boldsymbol{\Gamma} \boldsymbol{\eta}_{t-1},
$$

where and $\boldsymbol{B}=\operatorname{diag}\left(\boldsymbol{B}_{1}, \boldsymbol{B}_{1}\right)$ with $\boldsymbol{B}_{1}=$ $\left(\begin{array}{cc}0 & \frac{T}{n_{g}} \\ 0 & 0\end{array}\right)$. More sophisticated models can be considered to model targets' interactions in each group such as the developed in [19].

\section{B. Observation Model}

Range and bearing observations from a network of low cost sensors positioned along the road are considered as measurements. The measurement vector $\boldsymbol{z}_{t, i}$ for the $i$ th target contains the range $r_{t, i}$ to each target and the bearing $\beta_{t, i}$. The measurement equation is of the form:

$$
\boldsymbol{z}_{t, i}=h\left(\boldsymbol{x}_{t, i}\right)+\boldsymbol{w}_{t, i},
$$

where $h$ is the nonlinear function

$$
h\left(\boldsymbol{x}_{t, i}\right)=\left(\sqrt{x_{t, i}^{2}+y_{t, i}^{2}} \tan ^{-1} \frac{y_{t, i}}{x_{t, i}}\right)
$$

and the measurement noise $\boldsymbol{w}_{t, i}$ is supposed to be Gaussian, with a known covariance matrix $\boldsymbol{R}$.

\section{Particle Filtering Algorithms for Group Motion Estimation}

In this paper, two approaches are proposed. The impact of incorporating or not the group structure in the state is studied, also from the point of view of its computational complexity. One way of considering the group structure is to propagate, at each time step, a deterministic group structure using the previous group structure $\boldsymbol{G}_{t-1}$ and the current estimate of all the target states denoted by $\widehat{\boldsymbol{X}}_{t}$, i.e., $\boldsymbol{G}_{t}=f\left(\boldsymbol{G}_{t-1}, \widehat{\boldsymbol{X}}_{t}\right)$. Although the complexity of such an approach is reduced, it does not provide information about the group structure uncertainty. This group structure evolution model has been introduced in [29].

In contrast, by considering an augmented state (instead of $\boldsymbol{X}_{t}$, the state is now $\left(\boldsymbol{X}_{t}, \boldsymbol{G}_{t}\right)$ ), the group structure uncertainty can be accounted for in a better way. This group structure evolution model with an augmented state has been studied in [30]. In the next two subsections, particle filtering algorithms are presented combined with these two approaches.

1) Deterministic Update of the Graph Structure:

We denote by $N_{p}$ the number of particles and $L$ is the current index of a particle. Having in mind equations (2)-(5), the implemented algorithm is described as Algorithm 1, where the proposal PDF is of the form: $q_{g_{i}}\left(\boldsymbol{X}_{t}^{\boldsymbol{g}_{\boldsymbol{i}}} \mid \boldsymbol{X}_{0: t-1}^{\boldsymbol{g}_{i},(L)}, \boldsymbol{z}_{0: t-1}\right)=p\left(\boldsymbol{X}_{t}^{\boldsymbol{g}_{i}} \mid \boldsymbol{X}_{t-1}^{\boldsymbol{g}_{i},(L)}\right)$ (where $p\left(\boldsymbol{X}_{t}^{\boldsymbol{g}_{i}} \mid \boldsymbol{X}_{t-1}^{\boldsymbol{g}_{i},(L)}\right)$ is the transition PDF, for the target's state in the group $\boldsymbol{g}_{i}$, under the assumption that the interaction between targets is with respect to the group structure $\boldsymbol{G}_{t-1}$ ). In order to sample from this transition PDF, a nearly constant velocity model (6) is used for each component $\boldsymbol{X}_{t-1}^{\boldsymbol{g}_{i},(L)}$ of the particle $\boldsymbol{X}_{t-1}^{(L)}$ to obtain $\boldsymbol{X}_{t}^{\boldsymbol{g}_{i},(L)}$.

\section{JPDA Combined with the Estimated Group Structure} In step 2 of Algorithm 1, the data association problem is resolved by the JPDA algorithm [16]. The graph structure is used in the first step of the JPDA algorithm. Information contained in the graph structure is used to 
Algorithm 1. The particle filter with deterministic update of the group structure

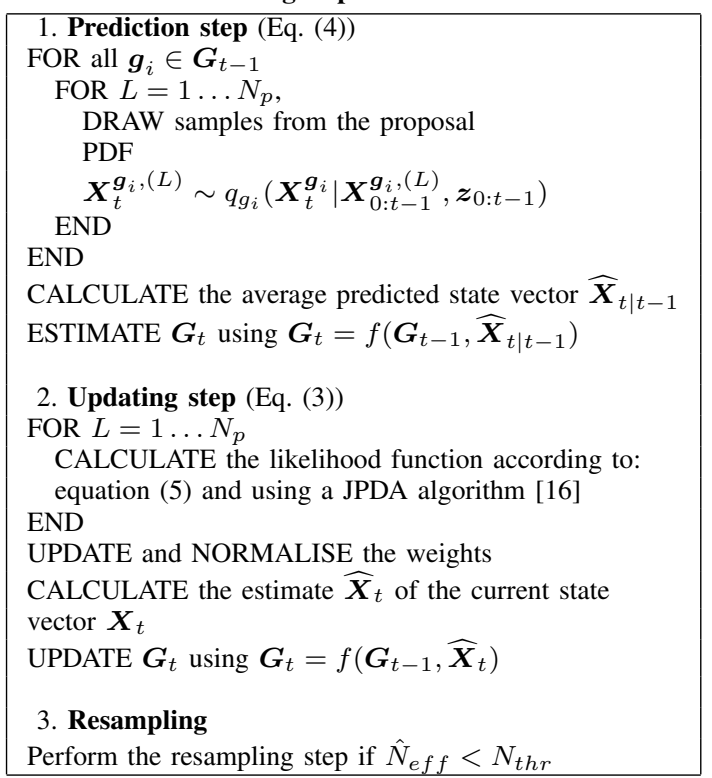

cluster the data association problem into distinct subproblems (Eq. (5)). This clustering stage helps reducing the computation time during the gating process (this gating process, in turn, is important for reducing the number of data associations hypotheses). The weight update is then performed by multiplying the likelihood by the previous time weights (Eq. (3))

Finally, in step 3 for each target we estimate the corresponding efficient components in the particles $\boldsymbol{X}_{t}^{(L)}$ and resample if the number of efficient particles $\hat{N}_{e f f}$ is less than a threshold $N_{t h r}: \hat{N}_{\text {eff }}<N_{t h r}$ [31].

2) Augmented State for a Graph Structure Uncertainty Estimation: In this Section we present a particle filtering technique with a Metropolis-Hastings (MH) step for group object motion estimation. Due to the augmented state with the graph structure, each particle contains the targets state and the group structure. In general, the MH steps are known to allow using less number of particles than the classical particle filter. We are, then introducing these $\mathrm{MH}$ steps in order to reduce the size of the particle cloud.

Having in mind (2)-(5), the implemented evolving group model is described as Algorithm 2, where the samples $\boldsymbol{X}_{t}^{\boldsymbol{g}_{i},(L)}$ are drawn from the proposal PDF $q_{g_{i}}^{(L)}\left(\boldsymbol{X}_{t}^{\boldsymbol{g}_{\boldsymbol{i}},(L)} \mid \boldsymbol{X}_{0: t-1}^{\boldsymbol{g}_{i},(L)}, \boldsymbol{z}_{0: t-1}\right)=p\left(\boldsymbol{X}_{t}^{\boldsymbol{g}_{i},(L)} \mid \boldsymbol{X}_{t-1}^{\boldsymbol{g}_{i},(L)}\right)$. The samples $\boldsymbol{G}_{t}^{(L)}$ for the graph structure are drawn from the PDF $Q\left(\boldsymbol{G}_{t} \mid \boldsymbol{X}_{0: t}, \boldsymbol{G}_{t-1}\right)=p\left(\boldsymbol{G}_{t} \mid \boldsymbol{X}_{t}, \boldsymbol{G}_{t-1}\right)$.

To sample from the proposal PDF $q_{g_{i}}$, a nearly constant velocity model (6)-(7) is used for each component $\boldsymbol{X}_{t-1}^{\boldsymbol{g}_{i},(L)}$ of a particle $\boldsymbol{X}_{t-1}^{(L)}$ to obtain $\boldsymbol{X}_{t}^{\boldsymbol{g}_{i},(L)}$.

The interactions within each group are modeled based on the mean velocity of group components (from the constant velocity model instead of the velocity of each group component).

To sample from the proposal PDF $Q$, the group struc-
Algorithm 2. Particle filtering with a state augmented by the group structure

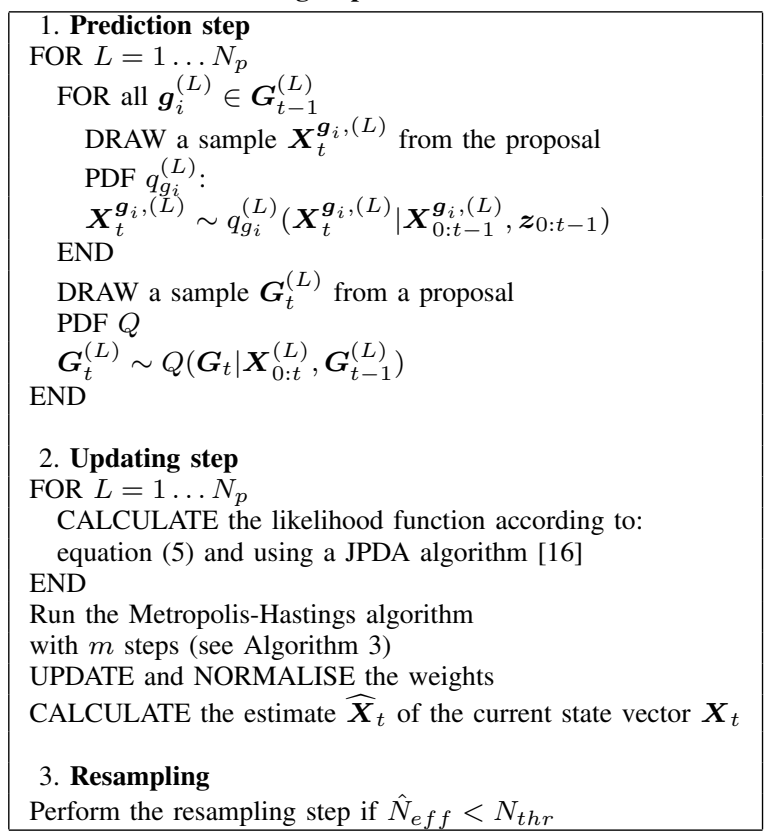

ture evolution model $\boldsymbol{G}_{t}=f\left(\boldsymbol{G}_{t-1}, \boldsymbol{X}_{t}\right)$ introduced in Section III-C, is used. In step 2 of Algorithm 2, the likelihood is calculated by assuming independence between clusters of measurements corresponding to each group. The MH step is described in Algorithm 3 and is iterated $m$ time steps ( $m$ being chosen beforehand). The MH algorithm is introduced to sample from the joint PDF $p\left(\boldsymbol{X}_{t}, \boldsymbol{G}_{t} \mid \boldsymbol{Z}_{1: t}\right)$.

In step 2 the likelihood and the weight update is performed, similarly to Algorithm 1, using the JPDA algorithm.

Finally, in step 3, for each target we estimate the corresponding efficient components in the particles $\boldsymbol{X}_{t}^{(L)}$ and resample if the effective number of samples $\hat{N}_{e f f}$ is less than a threshold $N_{t h r}$ [31].

Algorithm 3. Metropolis-Hastings step with the group structure

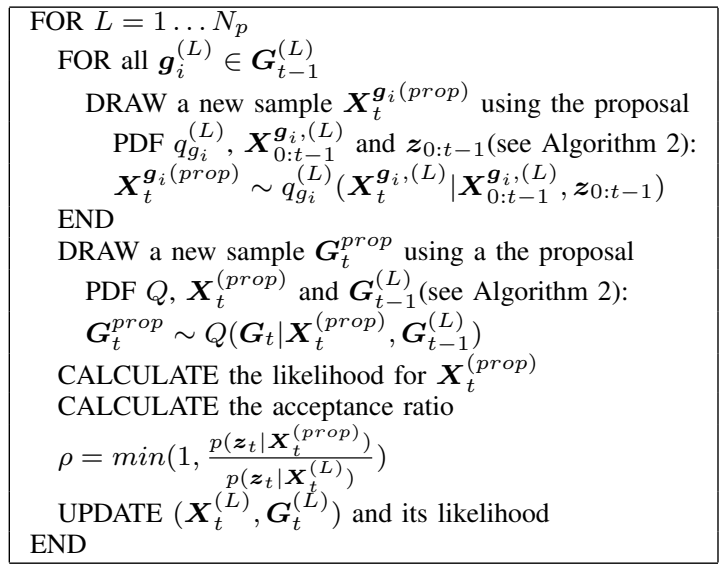

Note that, the particle filter presented in subsection IV-C1 can be implemented with a MH step, and respectively, the particle filter proposed in subsection IV-C2 can be implemented without the MH move step. 
Hence, we have four different types of algorithms. For conciseness, only two filters are presented in this paper. The next section contains simulation results and a comparison is made between the two presented particle filters.

\section{Simulation Results}

\section{A. Scenario and Models}

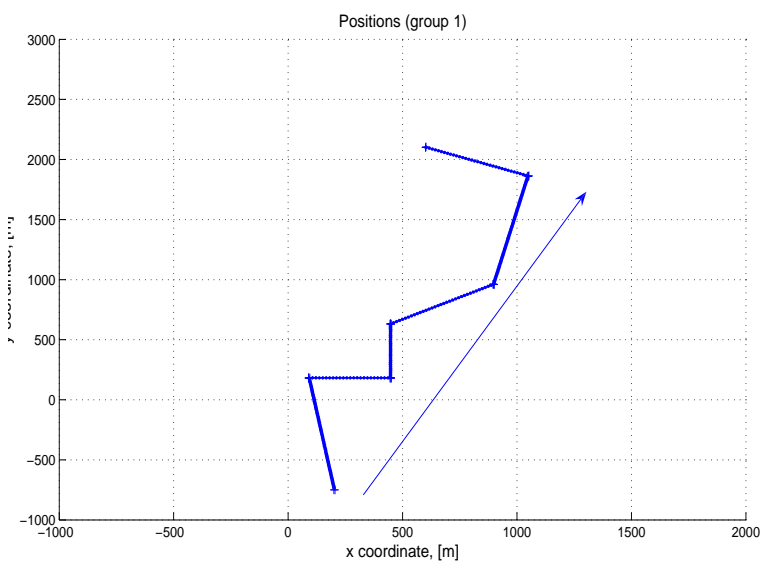

Fig. 6. Actual trajectory of group 1

The proposed techniques have been tested over a scenario in urban environment for ground moving object tracking. The movement of four groups (see Figures 6-9), each of them comprising two ground targets, is simulated over a period of $280 \mathrm{~s}$. All simulations and calculations have been done using a $4 \mathrm{GHz}$ Processor and Matlab software. The two filters provide outputs in every time second.

The scenario is the following: at the beginning, groups 1 and 2 form the same entity and split later in two groups during their motion. In contrast, groups 3 and 4 are two different entities at the beginning but merge into one group during the motion. In addition, group 1, during the time evolution, passes near groups 3 and 4 . Figure 10 shows this evolution of the group

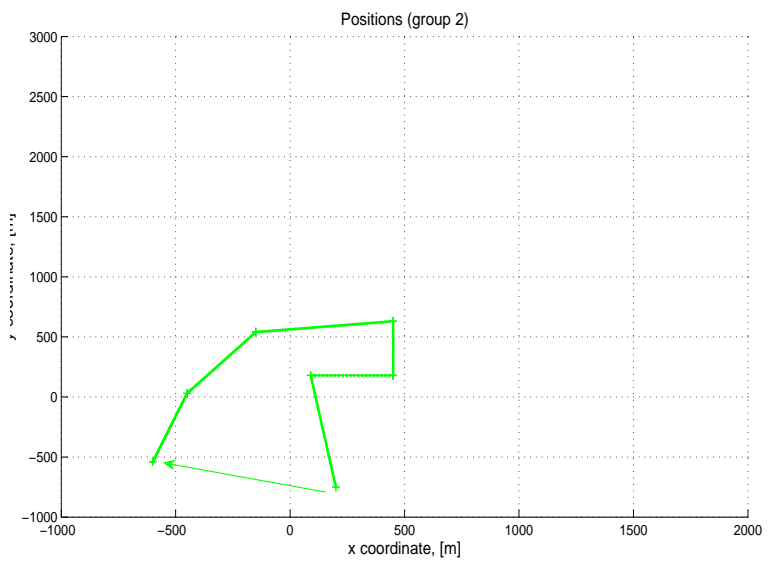

Fig. 7. Actual trajectory of group 2

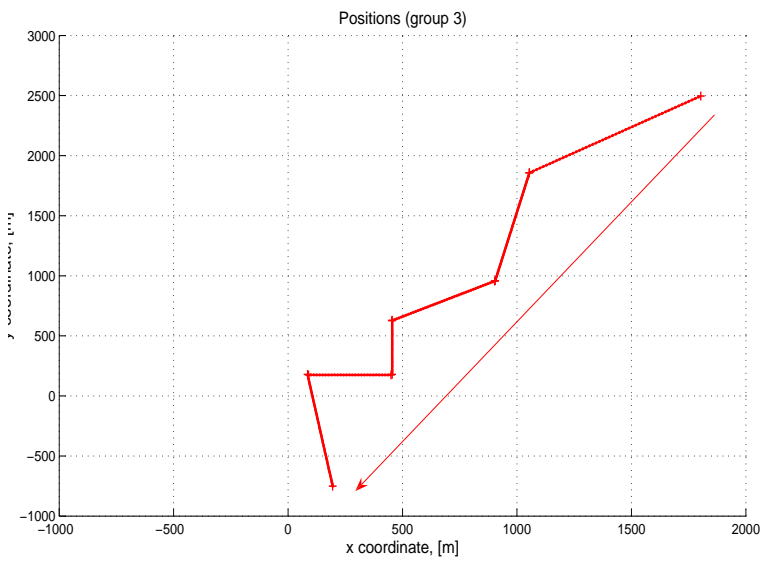

Fig. 8. Actual trajectory of group 3

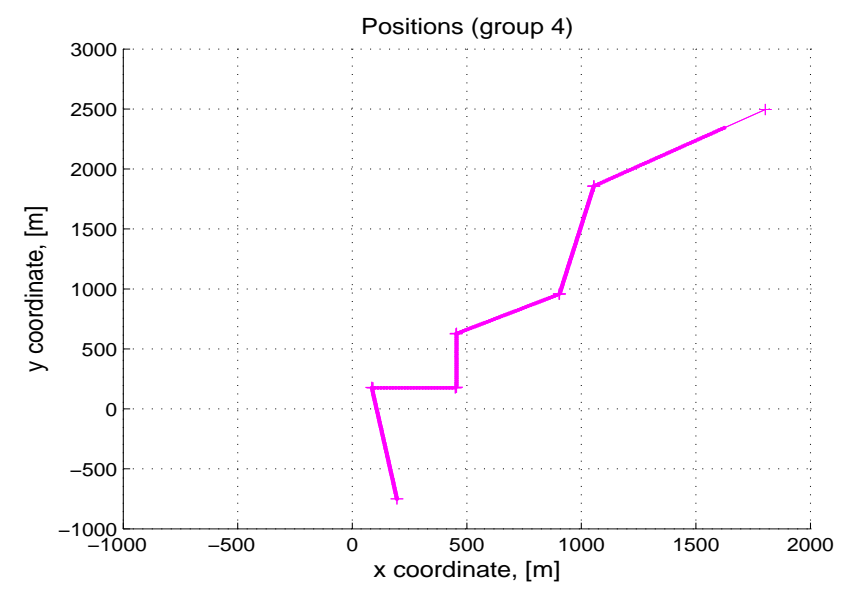

Fig. 9. Actual trajectory of group 4

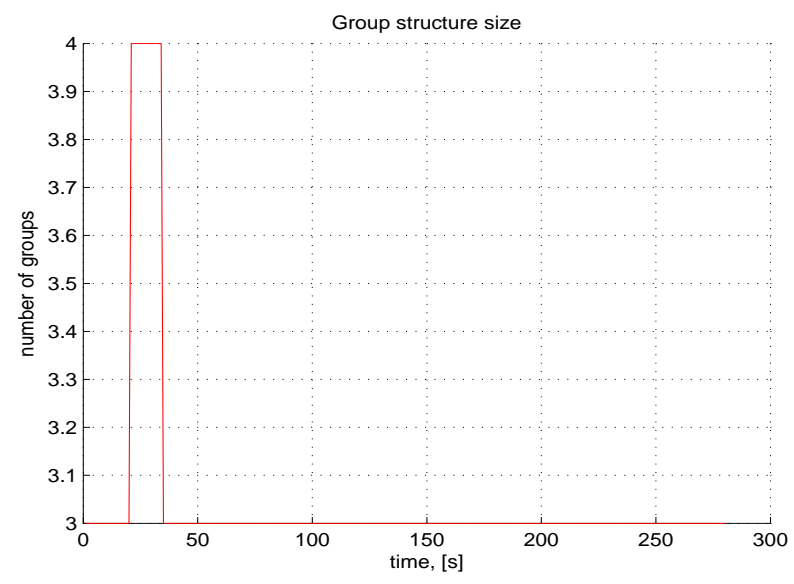

Fig. 10. Evolution of the group structure in time. From $0 s$ to $20 s$, three groups are evolving: $(1+2), 3$ and 4 . Then from time instant $20 \mathrm{~s}$ to $40 \mathrm{~s}$, four groups are evolving: $1,2,3$ and 4. Finally, from time instant $40 \mathrm{~s}$ to the end, three groups are evolving: 1,2 and $(3+4)$. 
structure with two changes due to, respectively, splitting and merging of groups.

The scenario is challenging since the filter should be able to deal with splitting and merging of groups and also should be able to avoid interactions with crossing groups coming from the opposite direction.

\section{B. Results}

1) Deterministic Group Structure Update: The particle filter $(\mathrm{PF})$ with sequential importance re-sampling (SIR) steps, described as Algorithm 1 in section IV-C1, has been applied to the previous scenario. Figures 1113 show the performance of the filter for all the 8 targets, 3000 particles and $N_{t h r}=300$. The coefficient $\alpha$ for the Gaussian sum in the constant velocity model has been chosen to be equal to 0.7 and the sampling interval is $T=1 s$. The Mahalanobis distance threshold for determining whether two targets are in the same group or not, has been chosen equal to respectively $55 \mathrm{~m}$ for the position and $15 \mathrm{~m} / \mathrm{s}$ for the velocity. These threshold values are very sensitive to the elements of the estimated covariance matrix for each target. A suitable choice of these parameters is necessary to avoid gatherings of two targets, with big difference in their speed or position, in the same group. The Mahalanobis distance threshold for the group centre has been chosen 4 times bigger than the previous thresholds.

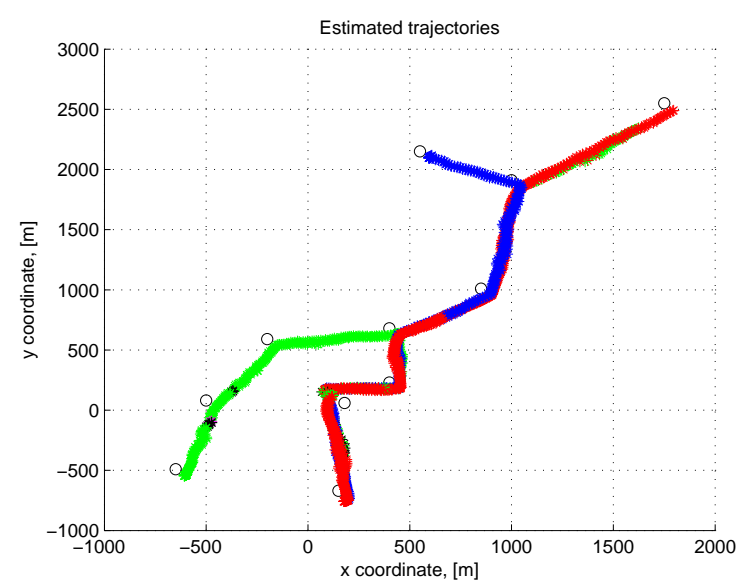

Fig. 11. Estimated trajectories for the 8 targets from a single run. The circles represent the sensor places.

\begin{tabular}{c||c|} 
& groups structures \\
\hline \hline 1 & $\boldsymbol{g}_{1}=\{1,2,7,8\} ; \boldsymbol{g}_{2}=\{3,4\} ; \boldsymbol{g}_{3}=\{5,6\}$ \\
\hline 2 & $\boldsymbol{g}_{1}=\{1,2\} ; \boldsymbol{g}_{2}=\{3,4,5,6\} ; \boldsymbol{g}_{3}=\{7,8\}$ \\
\hline 3 & $\boldsymbol{g}_{1}=\{1,2\} ; \boldsymbol{g}_{2}=\{3,4\} ; \boldsymbol{g}_{3}=\{5,6\} ; \boldsymbol{g}_{4}=\{7,8\}$ \\
\hline & \multicolumn{2}{||}{}
\end{tabular}

TABLE I

LISTING OF THREE GROUP STRUCTURES CORRESPONDING TO THE ACTUAL SIMULATED GROUP STRUCTURE EVOLUTION.

Figures 12 and 13 show the position mean errors from 50 Monte Carlo runs. The developed approach
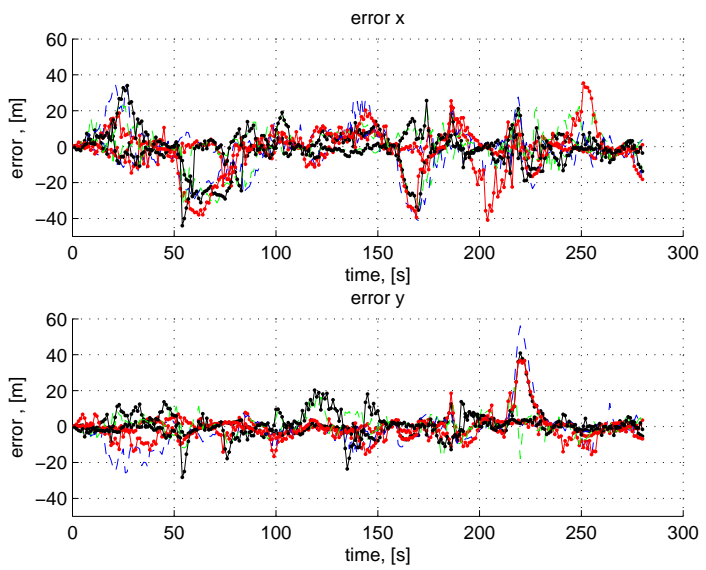

Fig. 12. Position estimation error for eight targets.
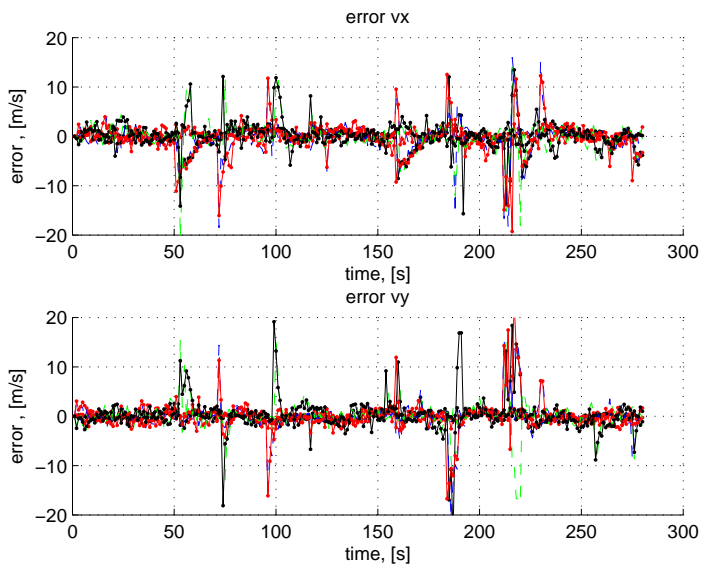

Fig. 13. Velocity estimation error for eight targets.

provides accurate estimates of the positions of the separate vehicles nevertheless a very simple first degree evolution model (constant velocity model) is used. Consequently, abrupt changes of velocity during the time evolution correspond to the spikes appearing on the Figures 16 and 17.

Figure 14 shows a comparison between the group structure evolution estimated using the $\mathrm{PF}$ and the group structure evolution estimated using the simulated trajectory. One can conclude that the group structure is well captured by the introduced graph evolution model. In addition, it is evident that the changes of the group structure are not detected at the same time instant due to the errors. The group structure estimated using the $\mathrm{PF}$ is also changing and incorporates five supplementary groups structures (4 to 8 ) different to the three ones presented in Table I. These supplementary group structures occur essentially when, due to estimation errors and during a short time, one group is abnormally split. Furthermore, the group crossing simulated in this scenario did not change the estimated group structure.

2) Augmented State with Group Structure: The SIR $\mathrm{PF}$ with the MH step has been applied to this scenario. Figures 15-17 show the performance of the filter for all the 8 targets. In this experiment 1000 particles have 

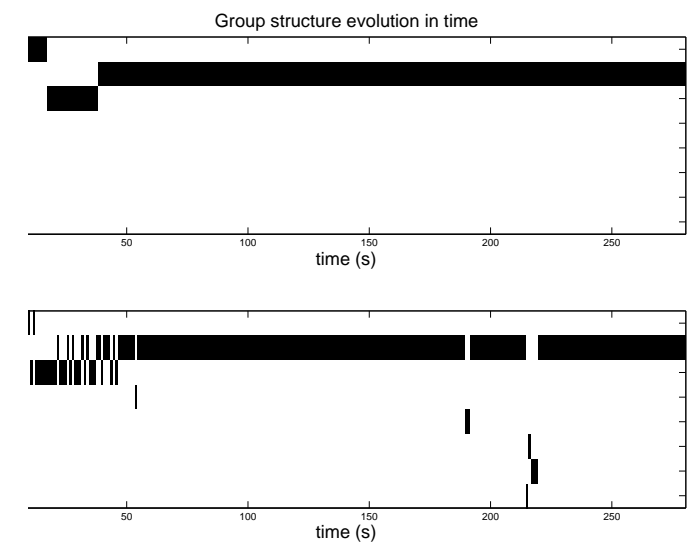

Fig. 14. Comparison between the group structure evolution estimated using the simulated trajectory (at the top) and using the PF (at the bottom)

been used with $m=10$ iteration of the $\mathrm{MH}$ algorithm. The coefficient $\alpha$ for the Gaussian sum in the constant velocity model has been chosen to be equal to 0.7 .

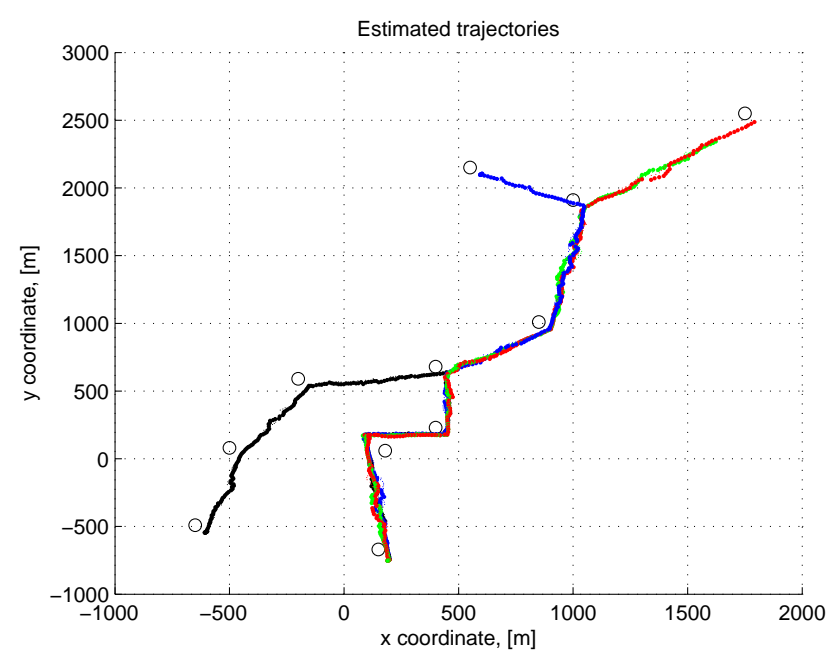

Fig. 15. Estimated trajectories for the 8 targets. the circles represent the sensor places.

Figures 16 and 17 show the position and velocity mean errors for 50 Monte Carlo runs. The developed approach provides accurate estimates of the positions of the separate vehicles. Additionally, Figure 18 presents the group structures estimated by the PF and the actual group structures of the simulated trajectories. The nine more relevant groups are labeled from 1 to 9 and a probability is calculated for each group at each time.

The group structures estimated by the $\mathrm{PF}$ give weights to five supplementary group structures (4 to 9). These supplementary group structures occur essentially when, due to estimation errors and during a short time, one group is abnormally split (especially when one edge is removed). Groups 4 to 6 differ from group 3, slightly, with one less edge and groups 7 to 9 differ
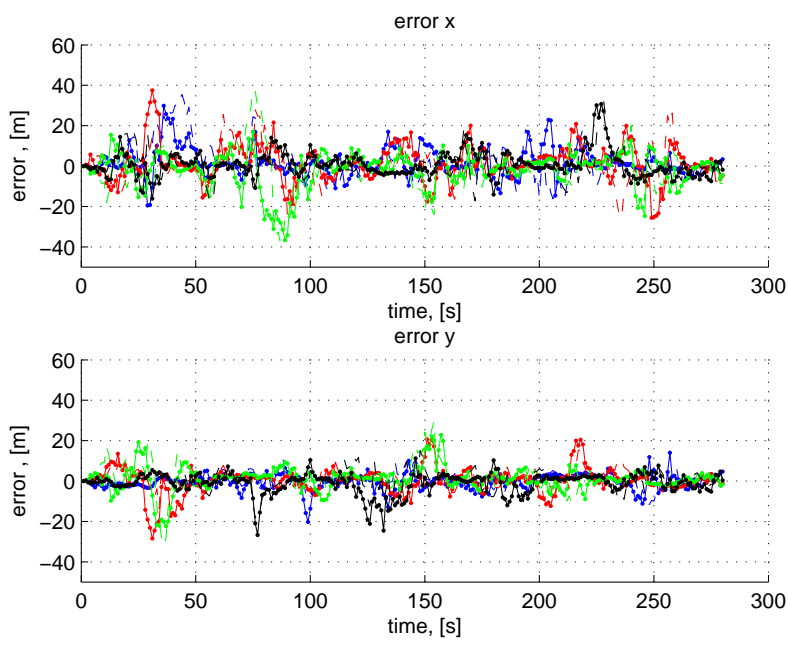

Fig. 16. Position estimation error for eight targets.
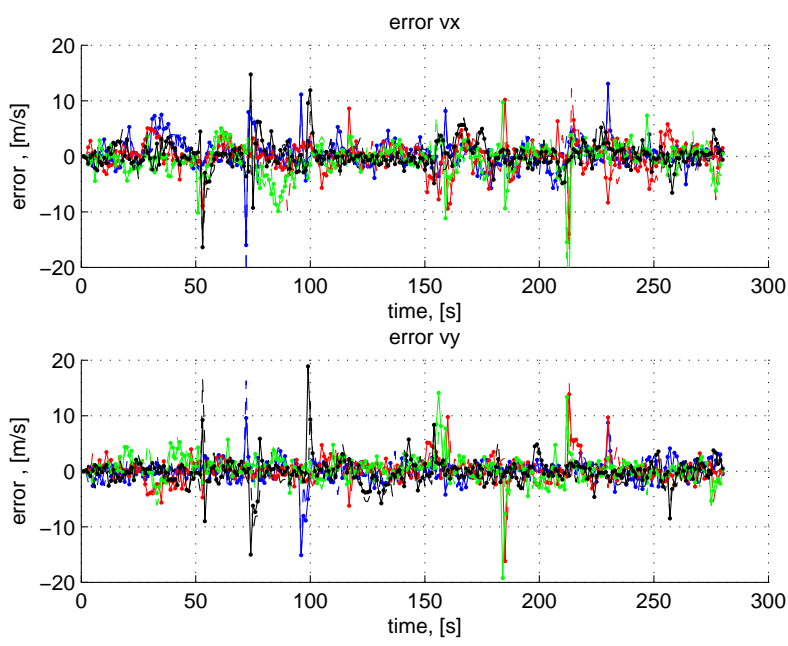

Fig. 17. Velocity estimation error for eight targets.

from group 2, slightly, with one less edge. One can conclude that the group structure is well captured by the introduced graph evolution model.

3) Comparison Between the Two Approaches: As expected, the PF with a state augmented by the group structure and incorporating an $\mathrm{MH}$ move step has shown slightly better accuracy that the PF with deterministic graph update. However, the computational complexity is increased substantially (approximately 3 times more) compared with the PF without the MH step and with deterministic update of the graph structure. The computational time for on time step (1s) are in average, respectively, $220 \mathrm{~ms}$ for the deterministic update and $500 \mathrm{~ms}$ for the second approach. Both approaches satisfy the real time constraint for these simulations with Matlab.

The approach with the augmented state can model well the group structure uncertainty and hence, gives more robust performance. 

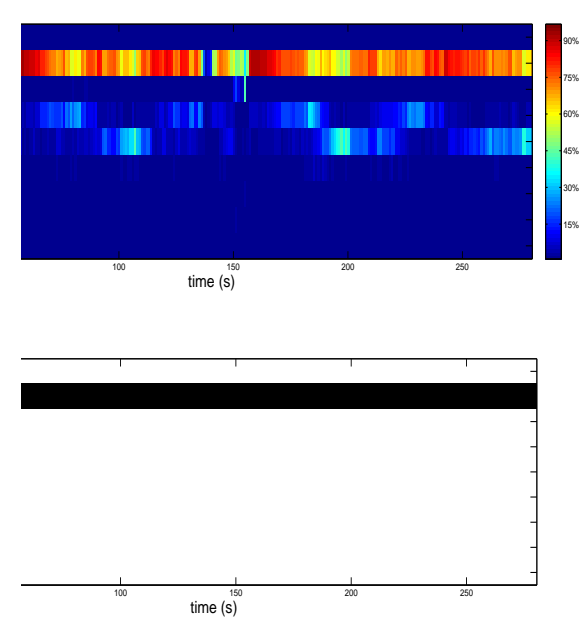

Fig. 18. Group structure evolution in the simulated trajectory (bottom) and estimated by the PF (top). The nine more relevant groups are labeled from 1 to 9 and a probability is calculated, for each group and at each time step, for the PF.

Nevertheless, that the application we consider is for groups of ground targets, the proposed techniques are quite general and can be applied to other systems, such as aircrafts or robots. In the next section, due to its robust performances and the possibility to provide group structure uncertainty, only the approach described on this section is applied to a real GMTI data set.

\section{RESUlts ON REAL DATA}
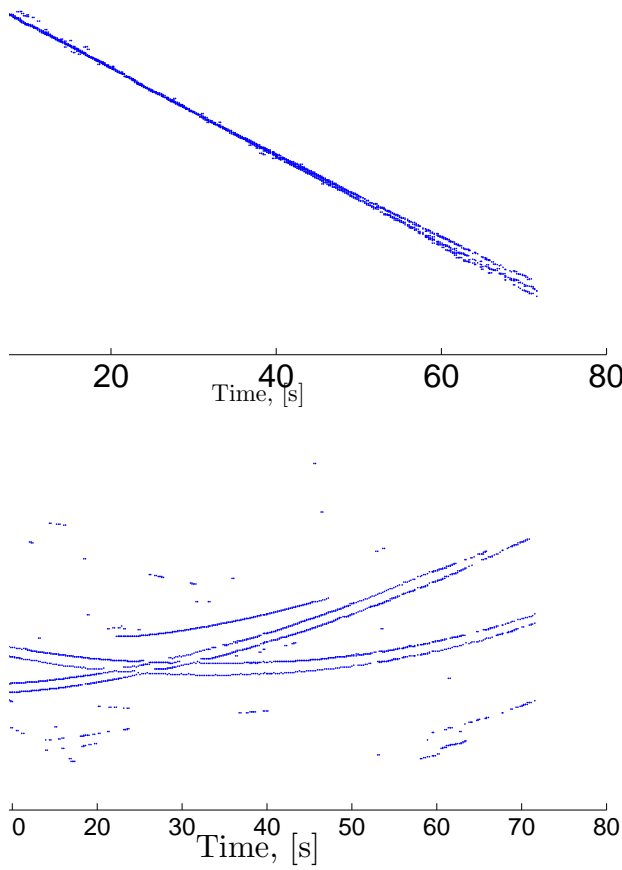

Fig. 19. Measured bearing and measured range, resp. for two groups.
This section presents results for the approach proposed in section V-B2. The validation is performed over real GMTI radar data shown in Figure VI provided to us by QinetiQ, UK. Two groups of targets are moving on the ground by crossing their paths which constitutes an additional ambiguity for the group tracking algorithm. The GMTI measurements are obtained by an embedded radar on a moving airborn platform. There is a measurement origin uncertainty which requires the solution of the data association problem.

As seen from Figure VI, there is clutter noise in the measured bearing angles and measured distances to the targets.

The developed approach provides good estimation accuracy of each vehicle trajectory positions (see Figure 20). Figure 21 shows additionally that the estimated $x$ coordinates of the groups are close to $x$ coordinates calculated from the measurements. The proposed algorithm is able to cope with the crossed trajectories of the groups.

\section{Trajectories}

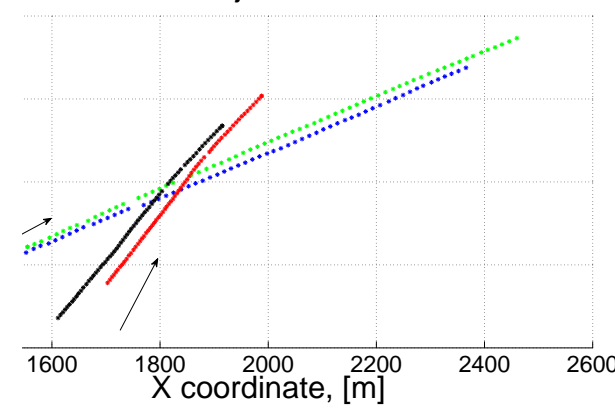

Fig. 20. Estimated trajectories for the 2 groups. The arrows show the directions of the movement

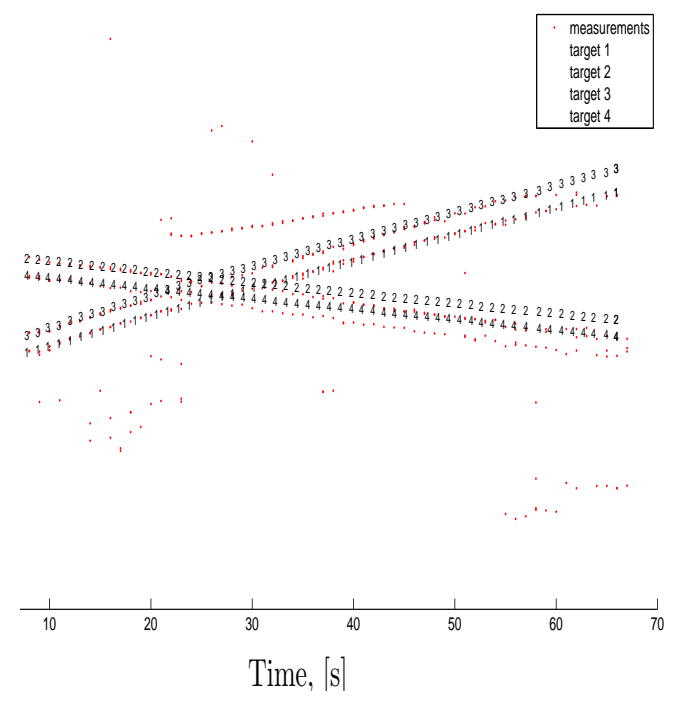

Fig. 21. This Figure shows the estimated $x$ coordinates for the 2 groups jointly with the $x$ coordinates are calculated from the measurements (converted from range and bearing). 
Figure 22 presents the group structures estimated by the particle filter. In the real scenario vehicles 1 and 2 are forming group 1 and vehicles 3 and 4 are forming the second group. To plot the Figure 22, only four relevant group structures appearing during the estimation process are labeled from 1 to 4 (respectively

$$
\begin{aligned}
& \boldsymbol{G}_{1}:\left\{\boldsymbol{g}_{1}=(1,2), \boldsymbol{g}_{2}=(3,4)\right\}, \\
& \boldsymbol{G}_{2}:\left\{\boldsymbol{g}_{1}=(1), \boldsymbol{g}_{2}=(2), \boldsymbol{g}_{3}=(3,4)\right\}, \\
& \boldsymbol{G}_{3}:\left\{\boldsymbol{g}_{1}=(1,2), \boldsymbol{g}_{2}=(3), \boldsymbol{g}_{3}=(4)\right\}, \\
& \boldsymbol{G}_{4}:\left\{\boldsymbol{g}_{1}=(1), \boldsymbol{g}_{2}=(2), \boldsymbol{g}_{3}=(3), \boldsymbol{g}_{4}=(4)\right\}
\end{aligned}
$$

and a probability is calculated for each group at each time. From Figure 22 one can conclude that the group structure is well estimated by the introduced graph evolution model. In addition, we can deduce precious information about the group structures uncertainty during the time evolution.

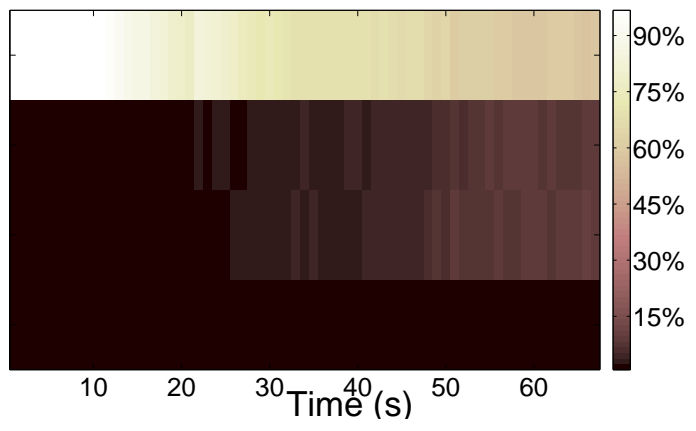

Fig. 22. Group structure evolution estimated by the PF. The 4 more relevant group structures are labeled from 1 to 4 (see (11)-(14)) and a probability is calculated, for each group and at each time step.

\section{CONCLUSIONS}

This paper presents Monte Carlo techniques for group object structure and state estimation. Evolutionary graph network-type models for the group structure are proposed. The graph structure can be deterministically estimated or in a probabilistic way with a graph jointly updated with the samples of the particle filter. The core idea is to maintain the structure of a graph in which connected components correspond to groups of targets. The effectiveness of the proposed techniques is investigated and validated over a challenging urban environment scenario with splitting, merging and crossing of groups. The performance of the approach is also validated over real ground moving target indicator data sets. The proposed approaches successfully estimate the targets states and the group structure graph with reliable performance and accurate tracking.

Acknowledgements. We are thankful the support from the EPSRC project EP/E027253/1, the Tracking Cluster project DIFDTC/CSIPC1 with the UK MOD
Data and Information Fusion Defence Technology Centre. We acknowledge the support from the [European Community's] Seventh Framework Programme [FP7/2007-2013] under grant agreement No 238710 (Monte Carlo based Innovative Management and Processing for an Unrivalled Leap in Sensor Exploitation) and the EU COST action TU0702. We appreciate also the help from QinetiQ, UK, including for providing us with the GMTI radar data, using RAISIN, QinetiQ's GMTI Processor and the QinetiQ PodSAR radar. We are thankful the Associate Editor and anonymous reviewers for their constructive suggestions to improve this paper.

\section{REFERENCES}

[1] M. Ulmke and W. Koch, "Road-map assisted ground moving target tracking," IEEE Trans. AES, vol. 42, no. 4, pp. 12641274, 2006

[2] A. Jadbabaie, J. Lin, and A. Morse, "Coordination of groups of mobile autonomous agents using nearest neighbor rules," IEEE Transactions on Automatic Control, vol. 48, no. 6, pp. $988-$ 1001, 2003.

[3] J. Marshall, M. Brouke, and B. Francis, "A pursuit strategy for wheeled-vehicle formations," in Proc. of 42nd IEEE Conf. on Decision and Control, Vol. 3, 2003, pp. 2555-2560.

[4] H. Axelsson, A. Muhammad, and M. Egerstedt, "Autonomous formation switching for multiple, mobile robots," in Proc. of the IFAC Conf. on Analysis and Design of Hybrid Systems, 2003.

[5] D. Dimarogonas and K. Kyriakopoulos, "A connection between formation control and flocking behaviour in nonholonomic multiagent systems," in Proc. IEEE International Conf. on Robotics and Automation, 2006.

[6] R. Mahler, Statistical Multisource-multitarget Information Fusion. Artech House, Boston, 2007.

[7] S. K. Pang, S. J. Godsill, F. Septier, J. Li, and S. Hill, "Sequential Inference for Dynamically Evolving Groups of Objects," Bayesian Time Series Models, edited by D. Barber, A.T. Cemgil and S. Chiappa (to be published), 2010.

[8] W. Konle, "Group tracking in an air surveillance system," in LNCS. Springer, 2009, pp. pp. 2434-2444.

[9] S. K. Pang, J. Li, and S. J. Godsill, "Detection and Tracking of Coordinated Groups," IEEE Transactions on Aerospace and Electronic Systems, Accepted, 2010.

[10] T. Vicsek, A. Czirók, E. Ben-Jacob, I. Cohen, and O. Shochet, "Novel type of phase transition in a system of self-driven particles," Phys. Rev. Lett., vol. 75, no. 6, pp. 1226-1229, Aug 1995.

[11] D. Helbing, "Traffic and related self-driven many-particle systems," Review of Modern Physics, vol. 73, pp. 1067-1141, 2002.

[12] M. J. Waxman and O. E. Drummond, "A bibliography of cluster (group) tracking," in Proceedings of the SPIE Signal and Data Processing of Small Targets, O. E. Drummond, Ed., vol. 5428, Aug. 2004, pp. 551-560

[13] S. N. Dorogovtsev and J. F. F. Mendes, "Evolution of networks," Advances in Physics, vol. 51, pp. 1079-1187, 2002.

[14] R. Albert and A.-L. Barabsi, "Statistical mechanics of complex networks," Reviews of Modern Physics, vol. 74, no. 1, pp. 4797, 2002.

[15] Y. Bar-Shalom and W. Blair, Multitarget-Multisensor Tracking: Applications and Advances. Boston: Artech House, 2000, vol. III.

[16] S. Blackman and R. Popoli, Design and Analysis of Modern Tracking Systems. Artech House Radar Library, 1999.

[17] D. Clark and S. Godsill, "Group target tracking with the Gaussian mixture probability density filter," in Proc. of the 3rd International Conf. on Intelligent Sensors, Sensor Networks and Information Processing, 2007.

[18] D. Clark, B. Vo, B.-N. Vo, and S. Godsill, "Nonlinear implementations of Gaussian mixture probability density filters," IEEE Transactions on Signal Processing, submitted, 2007. 
[19] S. K. Pang, J. Li, and S. Godsill, "Models and Algorithms for Detection and Tracking of Coordinated Groups," IEEE Aerospace Conf., March 2008.

[20] W. Koch and R. Saul, "A Bayesian approach to extended object tracking and tracking of loosely structured target groups," in Proc. of the 8th International Conf. on Inform. Fusion. ISIF, 2005.

[21] D. Salmond and N. Gordon, "Group and extended object tracking," in Proc. IEE Colloquium on Target Tracking: Algorithms and Applications, 1999, pp. 16/1 - 16/4.

[22] K. Gilholm, S. Godsill, S. Maskell, and D. Salmond, "Poisson models for extended target and group tracking," in Proceedings of SPIE 5913, 2005.

[23] B. Ristic, S. Arulampalam, and N. Gordon, Beyond the Kalman Filter: Particle Filters for Tracking Applications. Boston, London: Artech House, 2004.

[24] W. Koch and M. Feldmann, "Cluster tracking under kinematical constraints using random matrices," Robotics and Autonomous Systems, vol. 57, no. 3, pp. 296 - 309, 2009.

[25] W. Koch, "On exploiting 'negative' sensor evidence for target tracking and sensor data fusion," Inf. Fusion, vol. 8, no. 1, pp. 28-39, 2007.

[26] P. Erdös and A. Renyi, "On the evolution of random graphs," Publ. Math. Inst. Hung. Acad. Sci., vol. 5, pp. 17-61.

[27] X. R. Li and V. Jilkov, "A survey of maneuveuvering target tracking. Part I: Dynamic models," IEEE Trans. on Aerosp. and Electr. Systems, vol. 39, no. 4, pp. 1333-1364, 2003.

[28] Y. Bar-Shalom and X. Li, Estimation and Tracking: Principles, Techniques and Software. Artech House, 1993.

[29] A. Gning, L. Mihaylova, S. Maskell, S. K. Pang, and S. Godsill, "Evolving networks for group object motion estimation," in Proc. of IET Seminar on Target Tracking and Data Fusion: Algorithms and Applications, Birmingham, UK, 2008, pp. 99106.

[30] A. Gning, L. Mihaylova, S. Maskell, S. Pang, and S. Godsill, "Ground target group structure and state estimation with particle filtering," in Proc. of the 11th International Conf. on Information Fusion, Cologne, Germany, 2008, pp. 1176 - 1183.

[31] A. Doucet, N. Freitas, and E. N. Gordon, Sequential Monte Carlo Methods in Practice. New York: Springer-Verlag, 2001. 